\title{
Calibration procedures for approximate Bayesian credible sets: SUPPLEMENT
}

\author{
Jeong Eun Lee*, Geoff K. Nicholls ${ }^{\dagger, \S}$ and Robin J. Ryder ${ }^{\ddagger}$
}

\section{Appendix A: IS weight variance}

Denote by $\hat{d}$ the self normalised importance sampling estimate (of $d=d(y)$ ) returned by Algorithm 3. Recall that $d(y)$, defined in Equation 2.3, approximates $c(y)$ at small $\rho$, so using Algorithm 3 corresponds to choosing $\hat{c}=\hat{d}$. In this Appendix we prove that, for the application in Section 3, $\hat{d}$ satisfies a CLT, that is

$$
\sqrt{M}(\hat{d}-d) \rightarrow \mathcal{N}(0, V),
$$

with $V$ finite. We wish to estimate $d(y)$ where

$$
d(y)=\int_{\Omega} \int_{\mathcal{Y}} \mathbb{I}_{\phi \in \tilde{C}_{y^{\prime}}} m\left(\phi, y^{\prime}\right) d y^{\prime} d \phi
$$

and

$$
m\left(\phi, y^{\prime}\right)=z(y)^{-1} \pi(\phi) p\left(y^{\prime} \mid \phi\right) \mathbb{I}_{y^{\prime} \in \Delta_{y}}
$$

is a joint density with normalising constant $z(y)$. These are the expressions relevant for the application in Section 3. The $\theta$-integration has disappeared from $d(y)$ in Equation 2.4, because we have credible intervals $\tilde{C}_{y^{\prime}}$ which are exact for $\tilde{\pi}\left(\theta \mid y^{\prime}\right)$ in Section 3, not estimates $\hat{C}_{y^{\prime}}$ based on sampled $\theta$. Let $f\left(\phi, y^{\prime}\right)=\mathbb{I}_{\phi \in \tilde{C}_{y^{\prime}}}$ so that $d(y)=E_{m}\left(f\left(\phi, y^{\prime}\right)\right)$. Let $\tilde{m}\left(\phi, y^{\prime}\right)$ be the joint IS density with normalising constant $\tilde{z}(y)$,

$$
\tilde{m}\left(\phi, y^{\prime}\right)=\tilde{z}(y)^{-1} \tilde{\pi}(\phi \mid y) p\left(y^{\prime} \mid \phi\right) \mathbb{I}_{y^{\prime} \in \Delta_{y}} .
$$

Since $\tilde{\pi}(\phi \mid y)=\pi(\phi) \tilde{p}(y \mid \phi) / \tilde{p}(y)$, the normalised importance weight function $m / \tilde{m}$ is $w(\phi ; y)=k(y) / \tilde{p}(y \mid \phi)$ with $k(y)=\tilde{p}(y) \tilde{z}(y) / z(y)$. We now drop explicit $y$-dependence from notation. The unnormalised weight function is $\tilde{w}(\phi)=1 / \tilde{p}(y \mid \phi)$, so the unnormalised weights are for $i=1, \ldots, M, \tilde{w}_{i}=\tilde{w}\left(\phi_{(i)}\right)$ and our IS estimator is

$$
\hat{d}=\sum_{i} \tilde{w}_{i} c_{i} / \sum_{j} \tilde{w}_{j}
$$

with $c_{i}=f\left(\phi_{(i)}, y_{(i)}\right)$ and $\left(\phi_{(i)}, y_{(i)}\right) \sim \tilde{m}$ for $i=1, \ldots, M$.

\footnotetext{
* The Department of Statistics, University of Auckland, Private Bag 92019, Auckland 1142, New Zealand kate.lee@auckland.ac.nz

$\dagger$ Department of Statistics, 24-29 St Giles, Oxford, OX1 3LG, UK nicholls@stats.ox.ac.uk

$\ddagger$ CEREMADE, CNRS, Université Paris-Dauphine, PSL University, 75016 Paris, France ryder@ceremade.dauphine.fr

$\S$ Corresponding author.
} 
If posterior $\pi\left(\phi \mid y^{\prime}\right)=\pi(\phi) p\left(y^{\prime} \mid \phi\right) / p\left(y^{\prime}\right)$ is proper then $p\left(y^{\prime}\right)$ is finite at $y^{\prime}$. Since $\pi\left(\phi \mid y^{\prime}\right)$ in Section 3 is normal with finite non-zero variance and mean $y^{\prime} / 2$, it is proper at every $y^{\prime} \in \mathcal{Y}$. The same applies to $\tilde{\pi}\left(\theta \mid y^{\prime}\right)$ in Equation 3.2, so $p\left(y^{\prime}\right)$ and $\tilde{p}\left(y^{\prime}\right)$ are finite at each $y^{\prime} \in \mathcal{Y}$. It follows that $m$ and $\tilde{m}$ are proper densities. Also, $z, \tilde{z}$ and $k$ are finite, $E_{\tilde{m}}(w(\phi))=1$ and $d=d(y)$ itself is finite.

We now show that $\hat{d}$ has a CLT using the Multivariate Delta-Method. Let $\bar{d}=$ $M^{-1} \sum_{i} \tilde{w}_{i} k c_{i}$ and $\bar{w}=M^{-1} \sum_{i} k \tilde{w}_{i}$ denote normalised IS estimates, so that $\hat{d}=\bar{d} / \bar{w}$. Let $g(a, b)=a / b$, so that $\hat{d}=g(\bar{d}, \bar{w})$, with $g_{a}=1 / b$ and $g_{b}=-a / b^{2}$ partial derivatives. Let $\operatorname{var}(\bar{d})=U_{d} / M, \operatorname{var}(\bar{w})=U_{w} / M$ and $\operatorname{cov}(\bar{d}, \bar{w})=U_{d, w} / M$. If these quantities are all finite then by Theorem 5.5.28 of Casella and Berger (2002),

$$
\sqrt{M}(g(\bar{d}, \bar{w})-g(d, 1)) \rightarrow \mathcal{N}(0, V)
$$

as $M \rightarrow \infty$, with

$$
\begin{aligned}
V & =\left.\left(U_{d} g_{a}^{2}+2 U_{d, w} g_{a} g_{b}+U_{w} g_{b}^{2}\right)\right|_{(a, b)=(d, 1)} \\
& =U_{d}-2 d U_{d, w}+d^{2} U_{w}
\end{aligned}
$$

Now since $E_{\tilde{m}}(\bar{d})=E_{\tilde{m}}\left(f\left(\phi, y^{\prime}\right) w(\phi)\right)$ is equal $d$, we have

$$
\begin{aligned}
U_{d} & =E_{\tilde{m}}\left(\left(f\left(\phi, y^{\prime}\right) w(\phi)-d\right)^{2}\right) \\
& =E_{m}\left(f\left(\phi, y^{\prime}\right) w(\phi)\right)-d^{2},
\end{aligned}
$$

expanding the top line and using $E_{\tilde{m}}\left(f w^{2}\right)=E_{m}(f w)$ and $f^{2}=f$. The expectation in $\tilde{m}$ of $\bar{w}$ is 1 . Also, $U_{w}=E_{m}(w(\phi))-1$ (set $f, d$ equal 1 in $U_{d}$ ) and

$$
\begin{aligned}
U_{d, w}= & M E_{\tilde{m}}(\bar{d} \bar{w})-M d \\
= & M^{-1} \sum_{i} \sum_{j \neq i} E_{\tilde{m}}\left(f\left(\phi_{(i)}, y_{(i)}\right) w\left(\phi_{(i)}\right) w\left(\phi_{(j)}\right)\right) \\
& \quad+M^{-1} \sum_{i} E_{\tilde{m}}\left(f\left(\phi_{(i)}, y_{(i)}\right) w\left(\phi_{(i)}\right)^{2}\right)-M d \\
= & (M-1) d+M^{-1} \sum_{i} E_{m}\left(f\left(\phi_{(i)}, y_{(i)}\right) w\left(\phi_{(i)}\right)\right)-M d \\
= & E_{m}\left(f\left(\phi, y^{\prime}\right) w(\phi)\right)-d,
\end{aligned}
$$

where $E_{\tilde{m}}$ in the first term in the second line is expectation over independent pairs $\left(\phi_{(i)}, y_{(i)}\right),\left(\phi_{(j)}, y_{(j)}\right)$ each distributed according to $\tilde{m}$. Quantities $U_{d}, U_{w}$ and $U_{d, w}$ are finite if $E_{m}(w(\phi))$ is finite because $f \leq 1$ so $E_{m}\left(f\left(\phi, y^{\prime}\right) w(\phi)\right) \leq E_{m}(w(\phi))$, so $V$ in Equation 0.2 is finite ${ }^{1}$ and we are done.

In our setting, with $k$ and $z$ finite constants not depending on $y^{\prime}$ or $\phi$,

$$
E_{m}(w(\phi))=\int_{\Omega} \int_{\mathcal{Y}} \frac{k}{p(y \mid \phi)} m\left(\phi, y^{\prime}\right) d y^{\prime} d \phi
$$

\footnotetext{
${ }^{1}$ Substituting for $U_{d}, U_{w}$ and $U_{d, w}$ and using $E_{\tilde{m}}\left(f w^{2}\right)=E_{m}(f w)$ again gives $V=$ $E_{\tilde{m}}\left(w(\phi)^{2}\left(f\left(\phi, y^{\prime}\right)-d(y)\right)^{2}\right)$ the standard result (see for eg Owen (2013))
} 


$$
=\frac{k}{z} \int_{\Delta_{y}} \int_{\Omega} \pi(\phi) \frac{p\left(y^{\prime} \mid \phi\right)}{\tilde{p}(y \mid \phi)} d \phi d y^{\prime} .
$$

Since $k / z$ is finite, $E_{m}(w(\phi))$ is finite if $h\left(y^{\prime} ; y\right)=E_{\pi}\left(p\left(y^{\prime} \mid \phi\right) / \tilde{p}(y \mid \phi)\right)$ is bounded on the compact set $y^{\prime} \in \Delta_{y}$. For the densities we have in Section 3

$$
\begin{aligned}
h\left(y^{\prime} ; y\right) & \propto \int_{-\infty}^{\infty} \mathcal{N}(\phi ; 0,1) \exp \left(-\frac{\left(\phi-y^{\prime}\right)^{2}}{2}\right) \exp \left(\frac{v(\phi-y)^{2}}{2}\right) d \phi \\
& \propto \int_{-\infty}^{\infty} \exp \left(-\frac{\phi^{2}(2-v)}{2}+\phi\left(y^{\prime}-v y\right)\right) d \phi,
\end{aligned}
$$

which is finite for $0 \leq v<2$ and any fixed $y^{\prime}$.

We draw some general lessons. The IS estimator $\hat{d}$ for operational coverage $b(y)$ will have a CLT if the posteriors are proper and $E_{\pi}\left(p\left(y^{\prime} \mid \phi\right) / \tilde{p}(y \mid \phi)\right)$ is bounded for $y^{\prime} \in \Delta_{y}$. As usual in IS, problems arise when $\phi$-variation in the IS proposal (here the approximate posterior) is under-dispersed with respect to the IS target (here exact posterior), as this leads to large weight values. In our example things work well when $v$ is small (i.e. $0 \leq v<2)$ : when $0 \leq v<1$ the approximation is over-dispersed with respect to the exact posterior. When $v>1$ it is under-dispersed. There is some margin above $v=1$ due to the damping effect of the prior. If the approximation is under-dispersed then we might try Algorithm 2 (regression) ahead of Algorithm 3 (IS).

\section{Appendix B: Code}

Code generating the results in Sections 5, 6 and 7 is available in the online supplementary material (Lee et al., 2019).

\section{References}

Casella, G. and Berger, R. (2002). Statistical Inference. Duxbury advanced series in statistics and decision sciences. Thomson Learning, second edition. 2

Lee, J. E., Nicholls, G. K., and Ryder, R. J. (2019). "Supplementary material - code." URL https://github.com/robinryder/calibration 3

Owen, A. B. (2013). Monte Carlo theory, methods and examples, Chapter 9.

URL https://statweb.stanford.edu/ owen/mc/ 2 